\title{
Complications of Chemotherapy
}

\author{
Discussants: J.G. Cairncross, W. Pexman, M. Farrell \\ Edited by: J.J. Gilbert and J. Noseworthy
}

Can. J. Neurol. Sci. 1985; 12:149-153

\section{CASE REPORT}

A 2-year-old previously healthy girl presented to hospital because of irritability, fatigue, pallor and lower extremity weakness. Acute lymphoblastic leukemia (non-T non-B type) was diagnosed by peripheral blood smear and bone marrow aspirate. Chemotherapy was given and included vincristine, prednisone. L-asparaginase and intrathecal methotrexate. In addition, blood and platelet transfusions were given as appropriate. A lumbar puncture showed no cells, glucose $2.7 \mathrm{mmol} / \mathrm{L}$ (normal $2.2 \cdot 4.4 \mathrm{mmol} / \mathrm{L}$ ), protein of 0.40 (normal $0.150-0.450 \mathrm{~g} / \mathrm{L}$ ).

A routine chest $x$-ray had shown probable spinal column anomalies subsequently confirmed on thoraco-lumbar views as splitting of the TIO and TII vertebra with anterior fusion. Other examiners failed to demonstrate leg weakness and at discharge ( 5 weeks later) the child was walking normally.

Two weeks later, she returned for outpatient chemotherapy and received $0.65 \mathrm{mg} \mathrm{l} . \mathrm{V}$. vincristine and $5 \mathrm{mg}$ of intrathecal preservative free methotrexate. The CSF was clear and colourless with no cells, glucose of $2.8 \mathrm{mmol} / \mathrm{L}$, protein of $0.165 \mathrm{~g} / \mathrm{L}$. Bone marrow examination revealed a hypocellular remission phase picture.

Twenty-four hours later the child became irritable, drowsy, and hot. In the emergency room, the temperature was $38.4^{\circ} \mathrm{C}$; the child was irritable with opisthotonus. She complained of pain on neck and leg flexion. The cranial nerve examination showed equal pupils, reactive to light, normal external ocular movements, no facial asymmetry and a normal gag reflex. The upper extremities were considered normal but the lower extremities were described as weak with normal to reduced tone, absent knee and ankle reflexes and flexor plantar responses. Pinprick was appreciated in all extremities. The white blood count was $107 \times 10^{9} / \mathrm{L}$ (85\% neutrophils, $10 \%$ lymphocytes, $4 \%$ monocytes) with a moderate left shift and toxic granulations. CSF examination showed $124 \times 10^{6}$ white blood cells/l (83\% neutrophils. $7 \%$ lymphocytes, $10 \%$ monocytes), a glucose of $5.2 \mathrm{mmol} / \mathrm{L}$ and a protein of $0.250 \mathrm{~g} / \mathrm{L}$. Chloramphenicol and ampicillin were given but were subsequently changed to cloxacillin and moxalactam. CSF gram stain cultures and cytology were negative. Over the following 72 hours, the patient became more alert but remained febrile with opisthotonus and on one occasion, extensor plantar responses. However, on the 6th hospital day the level of consciousness deteriorated and there was further lower extremity flaccidity. The girl was febrile but rousible with opisthotonus. Fundi, pupils, and extraocular movements were normal and there was no facial asymmetry. The upper extremities moved in response to pain but the lower extremities were unresponsive and areflexic with absent plantar responses. The CSF showed $184 \times 10^{6}$ white blood cells/l $(83 \%$ neutrophils), $766 \times 10^{6}$ red blood cells, glucose of $2.8 \mathrm{mmol} / \mathrm{L}$ and a protein of $1.640 \mathrm{~g} / \mathrm{L}$. Gram stain and cultures were negative. Admission chemistry. WBC $-4.9 \times 10^{4} / \mathrm{L}$, hemoglobin $128 \mathrm{~g} / \mathrm{L}$, and platelet counts were normal. A computerized tomographic head scan was considered to be normal. An isotope brain scan showed abnormal diffuse patchy uptake over both cerebral hemispheres, and an EEG (Dysrhythmia Grade II - generalized) was considered compatible with "a moderate diffuse encephalopathy". Intravenous hydrocortisone was given and later replaced by dexamethasone. There was no improvement. The child developed frequent apneic spells lasting 6-8 seconds. A second CT scan showed enlarged ventricles and enlargement of the subarachnoid space. Folinic acid $(6.5 \mathrm{mg}$ in $6.5 \mathrm{ml}$ nonbacteriostatic normal saline) was administered intrathecally without benefit. CSF myelin basic protein was elevated at $8.9 \mathrm{mg} / \mathrm{L}$, and this was attributed to active demyelination. Increasing respiratory distress developed and she died on the thirteenth hospital day.

\section{Discussion}

\section{Clinical Features (Dr. Cairncross)}

This 2 year old girl with acute lymphoblastic leukemia (ALL) developed a fatal neurological illness, acute meningitis and an ascending flaccid quadriparesis. The neurological difficulties developed 24 hours after the intrathecal injection of methotrexate.

She presented with a typical picture of ALL. I suspect that her initial leg weakness was a non-specific finding related to the fatigue and malaise associated with a systemic disease. Other conditions must be considered including leukemic meningitis with infiltration of the cauda equina, leukemic infiltration of the lumbosacral plexus or peripheral nerves, spontaneous spinal subdural hematoma and pre-existing conditions such as an unsuspected spinal dysraphism or a neuromuscular disorder. Leukemic meningitis presents with headache, vomiting, mental change and diplopia, but rarely with symmetric lower extremity weakness. The normal lumbar puncture on the day chemotherapy was started excludes this diagnosis. Infiltration of the plexuses or peripheral nerves is an uncommon complication of acute leukemia and unlikely to be a presenting feature of the illness. The weakness was mild, symmetrical and confined to the lower extremities, features which speak against this diagnosis. Spinal subdural hematomas occur spontaneously in leukemic patients with profound thrombocytopenia. The absence of back pain and the essentially non-progressive nature of the leg weakness make this diagnosis unlikely. I cannot exclude an underlying spinal dysraphism and the abnormal plain $x$-ray films of the

From the Departments of Pathology (Neuropathology), Clinincal Neurological Sciences, and Radiology. University of Western Ontario Reprint requests to: Dr. J.J. Gilbert, Pathology (Neuropathology), Victoria Hospital, London, Ontario, Canada N6A 4G5 
lower thoracic spine lend some support to this possibility. Lastly, I have to consider an underlying congenital or familial neuromuscular disorder. There is very little to support this diagnosis.

I will briefly discuss the induction chemotherapy. At this centre patients receive vincristine $1.5 \mathrm{mg} / \mathrm{m}^{2}$ weekly for 4 weeks, L-asparaginase 10,000 units $/ \mathrm{m}^{2}$ intramuscularly three times a week for 6 doses, prednisone $20 \mathrm{mg} / \mathrm{m}^{2}$ for 4 weeks and intrathecal methotrexate $12 \mathrm{mg} / \mathrm{m}^{2}$ at induction and 4 weeks later at the time of the remission bone marrow. Vincristine can cause a peripheral, autonomic and cranial neuropathy and has been associated with inappropriate ADH syndrome. Vincristine neurotoxicity is dose related but occasional idiosyncratic reactions occur particularly in patients with pre-existing neuromuscular disorders. Curiously in our patient, the second course of vincristine was delayed for 7 days and the dose substantially reduced. I can only speculate that mild toxicity was observed following the first treatment. L-asparaginase produces an acute reversible encephalopathy developing within hours to days of administration. In this case the neurological difficulties began three weeks after the last dose of $\mathrm{L}$-asparaginase. The two common complications of intrathecal methotrexate are an acute self-limited chemical meningitis and a subacute leukoencephalopathy, seen after repeated courses of intrathecal methotrexate or high dose intravenous administration. Patients who have had cranial irradiation are more susceptible to the toxic effects of methotrexate on central white matter. This patient did not receive cranial irradiation. Lastly, patients taking steroids are prone to develop opportunistic CNS infections particularly if they are receiving chemotherapy and have an underlying disease which impairs immune mechanisms.

As an outpatient she received intrathecal methotrexate for the second time. The drug was reconstituted in a preservativefree solution. Twenty-four hours later there was fever, obtundation, prominent meningeal signs (findings typical of acute meningitis) and flaccid leg weakness. In some instances, areflexia and mild degrees of wasting and weakness of the legs follow bacterial meningitis as a consequence of adhesive arachnoiditis, but I believe the acute paraparesis must be explained on some other basis. I will consider the meningitis and the leg weakness independently. I suspect the two are related but it is not immediately obvious that this is so.

Acute bacterial meningitis is my first concern. In an otherwise healthy 2 year old, acute bacterial meningitis is usually due to Hemophilus influenza. However, this patient had leukemia, was receiving chemotherapy, was taking steroids and had had a recent lumbar puncture. Organisms other than $\mathrm{H}$. influenza must be considered; these include Staph. aureus, Strep. faecalis and enteric gram negative bacteria. The initial choice of ampicillin and chloramphenicol was reconsidered with cloxicillin and moxalactam being selected to provide a wider spectrum of coverage. I point out that cloxicillin and moxalactam do not cover pseudomonas aeruginosa. In a study of CNS infections in cancer patients at Memorial Sloan Kettering Cancer Centre ', there were no cases of bacterial meningitis among acute leukemia patients with normal peripheral white blood cell (WBC) counts. However, in leukopenic patients (WBC less than 2,700) there were a number of cases of acute bacterial meningitis, the majority due to pseudomonas. In our patient, the normal peripheral WBC, the repeatedly negative CSF cultures and the consistently normal CSF glucose levels speak against an acute or partially treated bacterial meningitis. Opportunistic CNS infections such as herpes zoster meningoencephalitis, cryptococcal meningitis and cerebral toxoplasmosis can be excluded on similar grounds. Leukemic infiltration of the leptomeninges is excluded by the presence of fever, normal lumbar puncture the preceding day and negative CSF cytology. I favour a severe chemical meningitis secondary to methotrexate.
The neurological examination pointed to a peripheral problem as the cause of the leg weakness. There were no spinal cord or cerebral signs. The differential diagnosis includes severe vincristine neuropathy, spinal subdural hematoma, leukemic meningitis with infiltration of the cauda equina and methotrexate neurotoxicity. I do not think the acute paraparesis was a toxic effect of vincristine. There is little evidence of an underlying neuromuscular disorder. Perhaps most importantly, I would expect some upper extremity findings with this degree of flaccidity, weakness and areflexia in the legs. In support of a spinal hematoma is the symmetric flaccid paraparesis appearing 24 hours after a lumbar puncture in a potentially thrombocytopenic patient but there is the absence of back pain and the platelet count was normal. Could intrathecal methotrexate have caused the acute paraparesis? The methotrexate was resuspended in a preservativefree solution. This is a key issue. Hahn et al ${ }^{2}$ reviewed all cases of acute paraparesis following intrathecal chemotherapy and reported a patient with meningeal lymphoma who developed sudden leg weakness following intrathecal cy tosine arabinoside. The authors concluded that preservative in the diluent was the likely explanation for the majority of cases of acute paraparesis following intrathecal chemotherapy. However, they noted several instances of sudden ascending flaccid paralysis and stupor leading to death in patients treated with preservative-free methotrexate solutions.

Four days after admission, the patient deteriorated. Neutrophils persisted in the CSF and the CSF protein increased. Neither the CT scan nor the EEG provided a diagnosis. The isotope brain scan showed diffuse patchy uptake over the hemispheres. This distribution of activity suggests destruction of the blood brain barrier in cortical vessels, a pattern one might see with fulminant meningitis.

To summarize, I believe this patient had a rare form of methotrexate neurotoxicity. Is there a precedent for this case? Bleyer et $\mathrm{al}^{3}$ described a patient who developed a flaccid ascending quadriparesis following intrathecal methotrexate. The patient died 17 days later. Neuropathological examination showed only scattered petechial hemorrhages throughout the brain and spinal cord. Skullerud and Halvorsen ${ }^{4}$ described a patient who developed a progressive flaccid quadriparesis and stupor after the fifth dose of intrathecal methotrexate. This patient had not received cranial irradiation and died 18 days after treatment. At autopsy, brain and spinal cord were grossly normal. Microscopic examination revealed superficial areas of necrosis and gliosis along the undersurface of the cerebral hemispheres, around the brainstem and cerebellum and along the entire spinal cord. Necrosis was severe at the root entry zones with loss of anterior horn cells and axons in ventral roots. The central white matter was normal in both the brain and cord. The meninges were normal although this patient had meningeal signs and a CSF pleocytosis 10 days prior to death. Ettinger ${ }^{5}$ reported a patient who died following an overdose of intrathecal methotrexate (50X the prescribed dose). The patient rapidly developed a flaccid areflexic quadriparesis with stupor and died 30 days later. There was no autopsy performed but CSF myelin basic protein was measured and found to be elevated (also previously reported by Clark et al ${ }^{6}$.

Williams et al ${ }^{7}$ recently reported ascending myeloencephalopathy due to intrathecal vincristine. The patient was a young boy with acute leukemia. He was to have received intrathecal methotrexate and intravenous vincristine but in error the syringes were interchanged. At autopsy there was extensive necrosis of all brain regions in contact with the CSF. I do not think our patient received intrathecal vincristine in error. Great care must be taken when administering these medications.

Dicussant's Diagnosis: Acute meningoencephalomyelitis, secondary to intrathecal methotrexate. 
Radiology (Dr. W. Pexman) The chest x-ray is normal. There is a mild kyphoscoliosis shown in the dorsolumbar region. The measurements of the interpedicular distances are not outside the normal range. Another radiologist had suggested that there may be some fused vertebral bodies but I think that this is an illusion produced by the scoliosis.

Computed tomography (CT) of the head was performed with and without contrast enhancement on an EMI 1010 scanner and shows no ventricular displacement, hemorrhage, no leukemic deposits, no abscess formation and no evidence of dural sinus thrombosis. There are no periventricular lucencies to suggest methotrexate leukoencephalopathy although it takes two months from the start of treatment for these to develop. The lateral ventricles are slightly enlarged for a two year old. The prominence of the temporal horns supports this assessment because on head CT displayed on a 256 pixel matrix the temporal horns are not seen in $90 \%$ of normal children or adults. The slight lateral ventricular dilatation may be due to some pre-existing atrophy possibly due to something that has happened earlier in the child's history such as foetal damage or perinatal or childhood trauma. Although cerebral atrophy is said to occur eventually in two-thirds of all children with acute lymphatic leukemia, it begins approximately eight months after the onset of the disease and treatment.

Four days later the second CT shows that the ventricles and the subaracahnoid spaces are slightly larger. This rapid change suggests that there is communicating hydrocephalus rather than atrophy and that the blockage of the cerebrospinal fluid pathway is at the level of the arachnoid granulations of the superior sagittal sinus. These could be affected by infective exudates or leukemic deposits and, as this second CT is unenhanced, we cannot exclude the possibility of a superior sinus thrombosis. However, as the cerebral sulci at the vertex have become more visible over four days it is most likely that the high CSF protein has produced communicating hydrocephalus in the present clinical context.

Pediatrics (Dr. L. deVeber) We have given approximately 1300 doses of intrathecal methotrexate over the years and have never seen anything like this. Children with leukemic meningitis treated by radiotherapy and intrathecal methotrexate can develop subacute CNS toxicity but we were not prepared for this type of reaction. This child was newly diagnosed and in remission with a $70 \%$ chance of cure. Children with acute leukemia frequently complain of pain in their legs and appear to have leg weakness. After the second course of intrathecal methotrexate she developed an acute meningitis with marked opisthotonus. There were abundant neutrophils in the CSF but no bacteria were seen on gram stain and none were grown. Although acute bacterial meningitis was the initial concern the neurological problems rapidly progressed in spite of antibiotics and it became clear that this was not a typical meningitis. Perhaps if we had made an initial diagnosis of methotrexate neurotoxicity earlier on we might have been able to prevent some of the toxicity by giving leucovorin intrathecally earlier. Washing out the CSF and systemic or intrathecal steroids may have been helpful. ${ }^{8.9}$ This is a recognized but extraordinarily rare complication of intrathecal methotrexate. On the basis of this case we have stopped giving intrathecal methotrexate routinely at three month intervals during the first two years of treatment.

Neuro-oncology (Dr. D. MacDonald) The rationale for giving intrathecal leucovorin in acute methotrexate toxicity is as follows: Folates are important in metabolism as single carbon donors. Dihydrofolates are converted to tetrahydrofolates, the actual carbon donors, by the enzyme dihydrofolate reductase. Methotrexate blocks this enzyme. In patients developing neurotoxicity from methotrexate prolonged and abnormally elevated levels of this drug have been demonstrated in the CSF. Leucovorin is given to bypass this metabolic block. Leucovorin given systemically does not penetrate the CNS and must be given directly into the intrathecal space. We used a preservative-free preparation of folinic acid. By this time the child was desperately ill and there was no clinical improvement.

Neurology (Dr. H.J.M. Barnett) The pediatricians have had great success in treating ALL. Dr. deVeber stated that on the basis of this experience he would no longer use prophylactic intrathecal methotrexate. Does he have an alternative medication or did I misunderstand him?

(Dr. L. deVeber) All patients receive prophylatic intrathecal methotrexate at the time of diagnosis and four weeks later at the time of the remission bone marrow. At this centre we have been giving additional intrathecal methotrexate at three month intervals for two years in an effort to further decrease the incidence of CNS leukemia. This extended series of treatments is optional and in view of this reaction we have altered our treatment plan. We are still giving prophylactic intrathecal methotrexate at the beginning of treatment.

(Dr. Barnett) Do you think this will compromise your cure rate?

(Dr. deVeber) No, there are studies to indicate that periodic intrathecal methotrexate treatments over an extended period do not increase survival.

Neuropathology (Dr. M. Farrell) The general autopsy did not reveal residual leukemia nor was there any septic focus nor evidence of bronchopneumonia. There was no compression of either spinal cord or cauda equina.

The brain had an unremarkable external appearance and the coronal sections were normal. Under the microscope multifocal areas of subpial astrocyte proliferation (Fig 1) located predominantly over the basal regions and to a lesser extent over both cerebral convexities were present. Subpial astrocytosis was particularly severe in the ventral midbrain but also involved

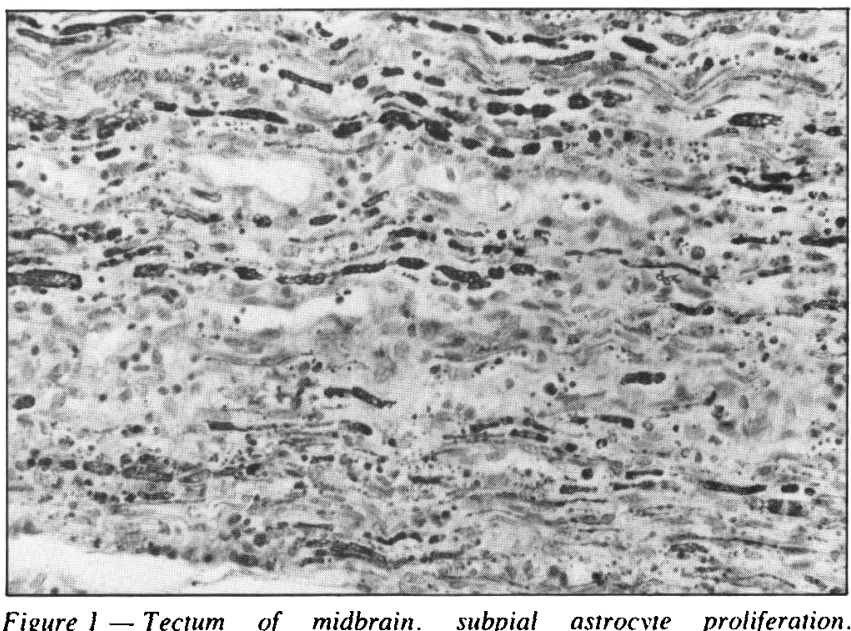

Figure I-Tectum of midbrain. subpial astrocyte proliferation. $H \& E, X 560$ 
the tectum and diencephalon. In all areas the pathological changes were superficial and in the cortex did not extend deeper than layer 3 . More severe and more acute changes were noted throughout much of the superficial cerebellar cortex. This necrotizing encephalopathy characterized by perivascular polymorphonuclear leukocytes, florid astrocyte proliferation, numerous macrophages and early cortical dissolution extended throughout much of the cerebellar molecular and superficial granular layers. Purkinje cells were shrunken and associated with marked pericellular vacuolation (Fig 2). The necrotizing process involved the superficial caudal brainstem close to the foramina of Luschka and also the floor of the fourth ventricle and overlying vermis.

The cerebral meninges showed a modest inflammatory cell infiltrate, mainly of macrophages with occasional fibroblasts. Polymorphonuclear leukocytes were not observed and there was no evidence of meningeal leukemia nor of any meningeal infection. The appearances were interpreted as resolving chemical meningitis.

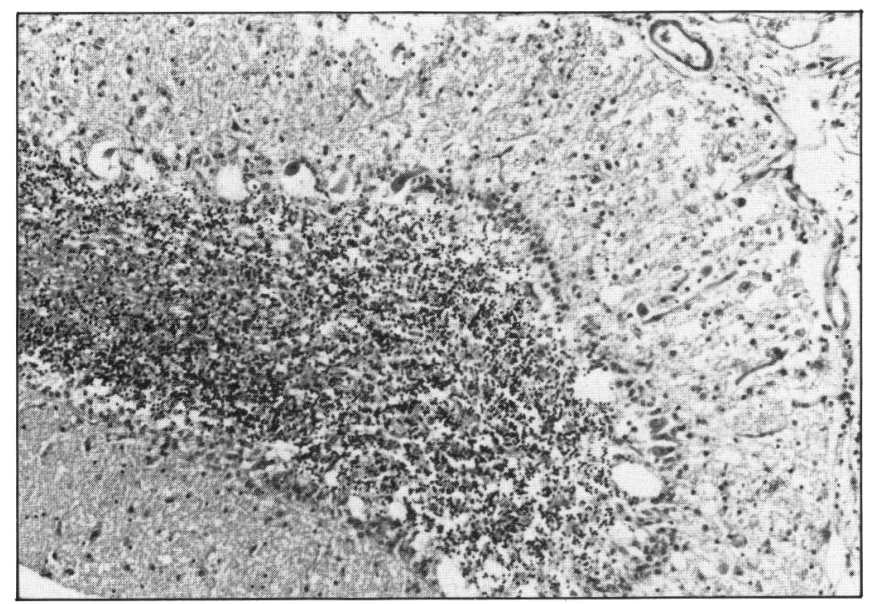

Figure 2 - Cerebellar folium. Necrosis of molecular layer. Loss of Purkinje cells. Infiltration by macrophages. $H \& E, X 560$.

Both hippocampi showed severe neuronal shrinkage with cytoplasmic eosinophilia indicative of an ischemic episode shortly before death.

The spinal cord was grossly normal. However, sections of the low thoracic and lumbar regions showed circumferential subpial myelin pallor which on high power examination consisted of vesicular disruption of the myelin sheath with axonal sparing. Striking changes were noted in dorsal and ventral roots, which showed severe axonal degeneration with concomitant myelin breakdown and myelin ovoid formation (Fig 3). The dorsal roots were affected disproportionately. Electron microscopy of spinal roots showed loss of axonal neurofilament organization progressing in some areas to complete loss of axoplasmic organelles and axolemma collapse (Fig 4). The lumbar spinal gray matter revealed chromatolysis of anterior horn cells.

In summary, the neuropathological diagnoses were as follows:

\section{Encephalopathy:}

a) cerebellar, necrotizing superficial cortical,

b) cerebral hemispheres, multifocal predominantly basal and involving brainstem and diencephalon.

2. Myelopathy, superficial, subpial, involving thoraco-lumbar white matter.

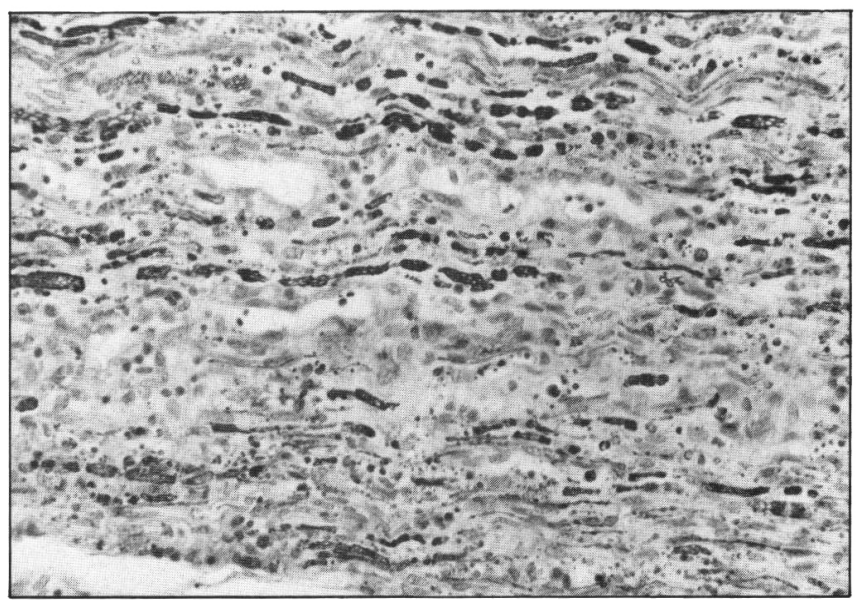

Figure 3 - Lumbar dorsal root. Wallerian degeneration, myelin fragments with macrophage infiltration. Solochrome $R$, myelin stain $X 560$.

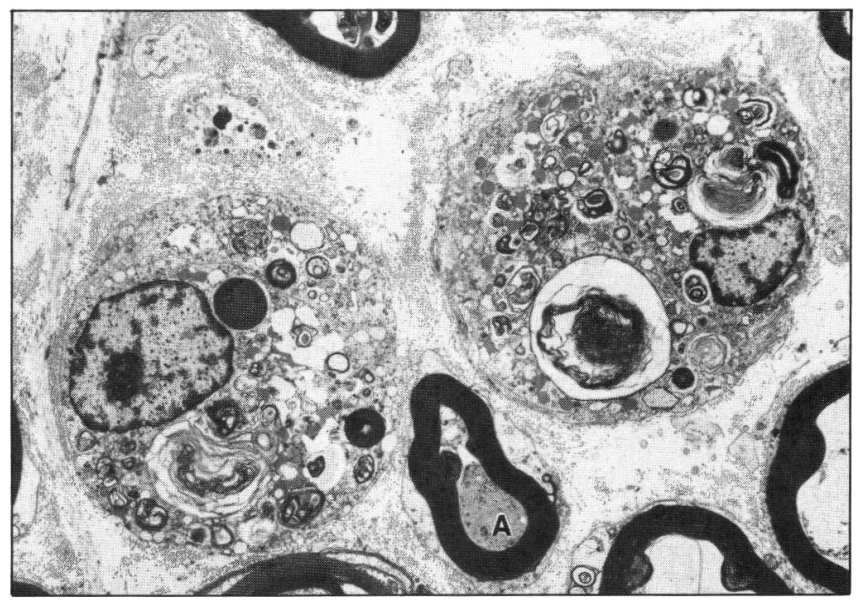

Figure 4-Lumbar spinal root. Schwann cells or macrophages filled with cellular debris, myelin figures. Other fibres show axonal condensation (A). EM. X 3000 .

3. Radiculopathy, sensory-motor, acute, severe, axonal with retrograde chromatolysis of anterior horn cells.

4. Meningitis, chemical, resolving, mild.

5. Ischemic encephalopathy, mild.

In a recent revie $w^{2} 18$ patients were reported to have developed paraparesis following intrathecal methotrexate, but in 10 of these it is possible to attribute the paraparesis to the neurotoxic effects of preservatives in either methotrexate or the associated diluent. Furthermore, many of the reported patients had received prophylactic cranio-spinal irradiation whilst in others there was insufficient pathological data to exclude neoplastic meningeal involvement, both possible alternative explanations for the paraparesis

The patient presented here is virtually unique in that the methotrexate and diluent were preservative-free. Cranio-spinal irradiation had not been previously administered and postmortem examination excluded meningeal leukemia factors which clearly directly implicate methotrexate as the causative agent. The striking subpial and radicular distribution of the pathological changes strongly suggests diffusion of neurotoxin from CSF into brain. An identical pattern of neurological involvement was noted by Skullerud and Halvorsen ${ }^{4}$ under similar circumstances. 


\section{ACKNOWLEDGEMENTS}

Publication of this clinical pathological conference of the University of Western Ontario, Department of Clinical Neurological Sciences has been made possible by generous support from Merck Frosst Canada Inc. and Sterling Products, a division of Sterling Drug Lid.

\section{REFERENCES}

1. Chernick NL, Armstrong D, Posner JB: Central nervous system infections in patients with cancer. Medi 1973: 52: 563-581.

2. Hahn AF, Feasby TE, Gilbert JJ: Paraparesis following intrathecal chemotherapy. Neurol 1983; 33: 1032-1038.

3. Bleyer WA, Drake JC, Chabner BA: Neuro-toxicity and elevated cerebrospinal fluid methotrexate concentration in meningeal leukemia. New Eng J Med 1973; 289: 770-773.
4. Skullerud K, Halvorsen K: Encephalomyelopathy following intrathecal methotrexate treatment in a child with acute leukemia. Cancer 1978; 42: 1211-1215.

5. Ettinger LJ: Pharmacokinetics and biochemical effects of a fatal intrathecal methotrexate overdose. Cancer 1982, 50: 444-450.

6. Clark AW, Cohen SR. Nissenblatt MJ. Welson SK: Paraplegia following intrathecal chemotherapy; neuropathological findings and elevation of myelin basic protein. Cancer 1982: 50: 42-47.

7. Williams ME, Walker AN, Bracikowski JP et al: Ascending myeloencephalopathy due to intrathecal vincristine sulfate: a fatal chemotherapeutic error. Cancer 1983; 51: 2041-2047.

8. Spiegel RJ, Cooper PR, Blum RH et al: Treatment of massive intrathecal methotrexate overdose by ventriculo-lumbar perfusion. New Eng J Med 1984; 311: 386-388.

9. Poplack DG: Massive intrathecal overdose "Check the label twice!". New Eng J Med 1984; 311: 400-401. 